\title{
Structural remodelling of cardiomyocytes in the border zone of infarcted rabbit heart
}

\author{
Ronald B. Driesen · Fons K. Verheyen · Petra Dijkstra • \\ Fred Thoné · Jack P. Cleutjens · Marie-Hélène Lenders • \\ Frans C. S. Ramaekers · Marcel Borgers
}

Received: 8 September 2006/ Accepted: 2 March 2007/Published online: 27 March 2007

(C) Springer Science+Business Media B.V. 2007

\begin{abstract}
Cardiomyocyte dedifferentiation, as detected in hibernating myocardium of chronic ischemic patients, is one of the characteristics seen at the border of myocardial infarcts in small and large animals. Our objectives were to study in detail the morphological changes occurring at the border zone of a rabbit myocardial infarction and its use as model for hibernating myocardium. Ligation of the left coronary artery (LAD) was performed on rabbit hearts and animals were sacrificed at $2,4,8$ and 12 weeks postinfarction. These hearts together with a non-infarcted control heart were perfusion-fixed and tissue samples were embedded in epoxy resin. Hibernating cardiomyocytes were mainly distributed in the non-infarcted region adjacent to the border zone of infarcted myocardium but only in a limited number. In the border zone itself vacuolated cardiomyocytes surrounded by fibrotic tissue were frequently observed. Ultrastructural analysis of these vacuolated cells revealed the presence of a basal lamina inside the vacuoles adjacent to the surrounding membrane, the presence of pinocytotic vesicles and an association with cisternae of the sarcoplasmatic reticulum. Myocyte
\end{abstract}

R. B. Driesen $(\bowtie) \cdot$ F. K. Verheyen · F. Thoné

M.-H. Lenders · F. C. S. Ramaekers - M. Borgers

Department of Molecular Cell Biology, Cardiovascular Research

Institute Maastricht (CARIM), University of Maastricht,

P.O. Box 616, 6200 MD Maastricht, The Netherlands

e-mail: r.driesen@molcelb.unimaas.nl

P. Dijkstra

Department of Animal Research and Testing Services,

University of Maastricht, P.O. Box 616,

6200 MD Maastricht, The Netherlands

\section{J. P. Cleutjens}

Department of Pathology, University of Maastricht,

P.O. Box 616, 6200 MD Maastricht, The Netherlands quantitative analyses revealed a gradual increase in vacuolar area/total cell area ratio and in collagen fibril deposition inside the vacuoles from 2 to 12 weeks post-infarction. Related to the remote zone, the increase in cell width of myocytes located in and adjacent to the border zone demonstrated cellular hypertrophy. These results indicate the occurrence of cardiomyocyte remodelling mechanisms in the border zone and adjacent regions of infarcted myocardium. It is suggested that the vacuoles represent plasma membrane invaginations and/or dilatations of $\mathrm{T}$-tubular structures.

Keywords Hibernation - Infarction - Border zone · Plasma membrane invaginations $\cdot$ T-tubules $\cdot$ Vacuoles

\section{Introduction}

The border zone of a myocardial infarction can be conceptualized as ischemically damaged though viable myocardium adjacent to an evolving myocardial infarction [1]. At the border zone of infarcted myocardium, viable cardiomyocytes (CM's) undergo drastic reorganization of cellcell and cell-extracellular matrix interactions. They lose cell-cell connections because of the death of neighbouring cardiomyocytes and subsequent anchoring to scar tissue [2]. Furthermore, it has been shown that the border zone of infarcted myocardium in larger animals such as dog and sheep may comprise a considerable number of dedifferentiated CM's, which are phenotypically similar to those seen in chronic hibernation [3-5]. Partial dedifferentiated cardiomyocytes have recently been described as well in the border zone of rat myocardium after myocardial infarction [6]. Hibernation of the myocardium was originally described as a protective mechanism of the heart in which it 
down regulates its contracting function to cope with oxygen shortage, caused by a chronic or repetitive underperfusion accompanied by a limited flow reserve [7]. The most obvious subcellular changes in these dedifferentiated CM's were described later on and comprised sarcomere depletion, glycogen accumulation, nuclear heterochromatin redistribution, mitochondrial shape changes and sarcoplasmic reticulum breakdown, giving the CM's a fetal/ neonatal-like phenotype [3]. After revascularisation, the hibernating segments often show a delayed recovery of function which can take a few months to more than one year in a fraction of patients with chronic hibernating myocardium [8]. It has been suggested that the structural remodelling found in chronic hibernating myocardium is at least partially responsible for this delayed recovery of function [9-11]. In recent studies we have also characterized the dedifferentiation process in an in vitro model by co-culturing adult rabbit CM's with cardiac fibroblasts [12, 13]. These studies showed large similarities in structural characteristics of hibernation-like dedifferentiation between rabbit and human CM's. As shown in previous reports, the rabbit heart appears to be similar in certain aspects to the human heart in that it presents a minor network of collateral coronary vessels under physiologic conditions $[14,15]$. Moreover, a histopathological study of the evolution of myocardial infarction without reperfusion in rabbit hearts also showed similarities to the human situation [15]. Therefore, to find out whether the rabbit heart can be used as a small animal model system for detailed studies on hibernation-like structural adaptations in CM's, myocardial infarcts were induced by coronary artery ligation, giving special attention to the infarct border zone.

The specimens were examined by light and electron microscopy and showed the presence of only a limited amount of hibernating myocardial cells. Obviously, CM's with apparent vacuolization were observed in the border zone of infarcted myocardium.

\section{Materials and methods}

All experimental procedures and protocols were carried out according to the Dutch law on animal experimentation and approved by the Animal Experimental Committee of Maastricht University. The investigation conforms to the Guide for the Care and Use of Laboratory Animals published by the US National Institutes of Health (NIH Publication No. 85-23 revised 1996).

Drugs and chemicals

Nimatek ketamine was purchased from Eurovet Animal Health (Bladel, NL), Diazepam from Centrafarm BV
(Etten-Leur, NL) and Forene isoflurane from Abbott BV (Hoofddorp, NL). Pentrexyl ampicilline was purchased from Bristol-Myers Squibb BV (Woerden, NL) and Temgesic buprenorphine from Schering-Plough BV (Utrecht, NL).

Veronal acetate, Toluidin Blue $\mathrm{O}$ and HEPES were obtained from Sigma Chemical (St Louis, USA). Epoxy resin and uranyl acetate were purchased from LADD Research industries (Vermont, USA) and osmium tetroxide was from Electron Microscopy Services (Hatfield, UK). Glutaraldehyde, lead citrate, periodic acid Schiff and the KRH-buffer substitutes were purchased from Merck (Darmstadt, Germany).

Experimental animals

\section{Development of coronary ligation model}

In four New Zealand rabbits, weighing between 2 and $3 \mathrm{~kg}$, a myocardial infarction was induced by ligation of a branch of the left anterior descending coronary artery (LAD). The rabbits were anaesthetized with Ketamine $(15 \mathrm{mg} / \mathrm{kg}$, i.m.) and Diazepam $(0.5 \mathrm{mg} / \mathrm{kg}$, i.m.). After endotracheal intubation, the rabbits were ventilated with a mixture of $\mathrm{O}_{2}$ and $\mathrm{N}_{2} \mathrm{O}$ (1:2) and 1.5-2.5\% isofluran, whereafter an intramuscular injection of ampicilline $(250 \mathrm{mg} / \mathrm{kg})$ was given. A twelve lead electrocardiogram was recorded throughout the operation. A left thoracotomy was made through the fifth intercostal space and the pericardium was opened. To produce an anterior myocardial infarction, a distal branch of the LAD was selected for ligation. A suture was placed around the vessel, which could be tightened by a silicon tube. The selected artery was temporarily occluded and the extent of ischemia was visually and electrocardiographically assessed for $15 \mathrm{~min}$, whereafter the artery was permanently ligated. After the surgical procedure, the chest was closed and $0.04 \mathrm{mg} / \mathrm{kg}$ buprenorfine was administered by intramuscular injection.

\section{Measurement of the electrocardiogram}

A standard ECG was recorded under anesthesia at a chartspeed of $25 \mathrm{~mm} / \mathrm{s}$. A successful induction of myocardial infarction was confirmed by elevation of the ST segment by more than $0.2 \mathrm{mV}$ in leads I, II and aVL.

\section{Fixation by Langendorff perfusion}

Four rabbit hearts at 2, 4, 8 and 12 weeks after induction of the myocardial infarction and one rabbit heart without ligation were excised and immediately perfused during 5 min via the aorta with a modified Krebs-Ringer-Henseleit buffer $\left(120 \mathrm{mM} \mathrm{NaCl}, 2.5 \mathrm{mM} \mathrm{KCl}, 1.5 \mathrm{mM} \mathrm{KH}{ }_{2} \mathrm{PO}_{4}\right.$, 
$1.2 \mathrm{mM} \mathrm{MgSO}{ }_{4} \cdot 7 \mathrm{H}_{2} \mathrm{O}, 10 \mathrm{mM}$ HEPES, $9.5 \mathrm{mM}$ glucose and $5 \mathrm{mM}$ pyruvate) to rinse the blood out of the coronary blood vessels. Subsequently, the heart was fixed by perfusion with $3 \%$ glutaraldehyde buffered to $\mathrm{pH} 7.4$ with $90 \mathrm{mM} \mathrm{KH}_{2} \mathrm{PO}_{4}$ for $5 \mathrm{~min}$.

\section{Histopathology and ultrastructure}

After perfusion fixation, small biopsies measuring $2 \mathrm{~mm}$ in diameter were obtained from the border zone of the infarcted area and immersion fixed for at least $24 \mathrm{~h}$ with $3 \%$ glutaraldehyde buffered to $\mathrm{pH} 7.4$ with $90 \mathrm{mM} \mathrm{KH} \mathrm{KO}_{4}$. Thereafter, the samples were washed in the same buffer for $24 \mathrm{~h}$ and post-fixed for $1 \mathrm{~h}$ in $2 \% \mathrm{OsO}_{4}$ buffered to $\mathrm{pH} 7.4$ with veronal acetate. Next, the samples were rapidly dehydrated through a graded ethanol series, and routinely embedded in Epon. Semi-thin sections were stained with periodic acid Schiff (PAS) and/or toluidin blue for light microscopical evaluation. Ultra-thin sections were counterstained with uranyl acetate and lead citrate prior to examination in a Philips CM100 electron microscope.

\section{Quantitative evaluation}

For morphometric analysis of the infarcted and non-infarcted myocardial light microscopical sections, a Leica DM 5000B microscope equipped with a Leica DC 300 FX camera was used in combination with a computerized surface determination method i.e. Leica QWin morphometry software v. 3.2.1. (Leica, Cambridge Instruments, Ltd., Cambridge, UK) that employed onscreen visualization of the cardiac sections. Cell width of CM's was measured in the border zone of infarcted myocardium, in the adjacent non-infarcted region (limited to a thickness of eight cell layers) and in the remote zone of the infarcted hearts; in the non-infarcted control heart an area beneath the level of the ligation site was selected. Only CM's containing a recognizable nucleus were analyzed for this parameter. Vacuole area and total cell area was measured in CM's surrounded by fibrotic tissue in the border zone of infarcted myocardium. The ratio of the vacuole area versus the total cell area was calculated and expressed as percentage.

Collagen fibril deposition in vacuoles of border zone CM's was quantitatively evaluated in the electron microscope. The ratio between the amount of positive vacuoles and the total number of vacuoles per cell was calculated and expressed as percentage.

\section{Statistical analysis}

Data are reported as mean \pm standard deviation. Statistical significance was indicated by $P<0.05$. Regional differences between groups were compared by one-way analysis of variance (ANOVA) for multiple comparisons. Bonferroni post hoc testing was used to determine the significance of the differences between the various groups.

\section{Results}

Characterization of the infarct border zone

Macroscopically, ligation of a distal branch of the LAD resulted in a relatively small, near transmural infarct in the apical part of the left ventricle (Fig. 1). Tissue sections taken from this region were stained by PAS and/or toluidin blue and examined in the light microscope. The three regions of interest were: (1) the border zone of infarcted myocardium in which several transections of CM's could be found which were surrounded by fibrotic scar tissue, (2) the adjacent non-infarcted region and 3 ) the (non-infarcted) remote zone (Figs. 1 and 2a, b). In the samples taken from remote zones and the adjacent non-infarcted regions, fibrotic tissue was not observed and the CM's showed normal mutual associations with each other and with the intermingled microcirculatory blood vessels (Fig. 2a, b). Figure 3a shows the normal ultrastructural morphology of a CM from a remote zone.

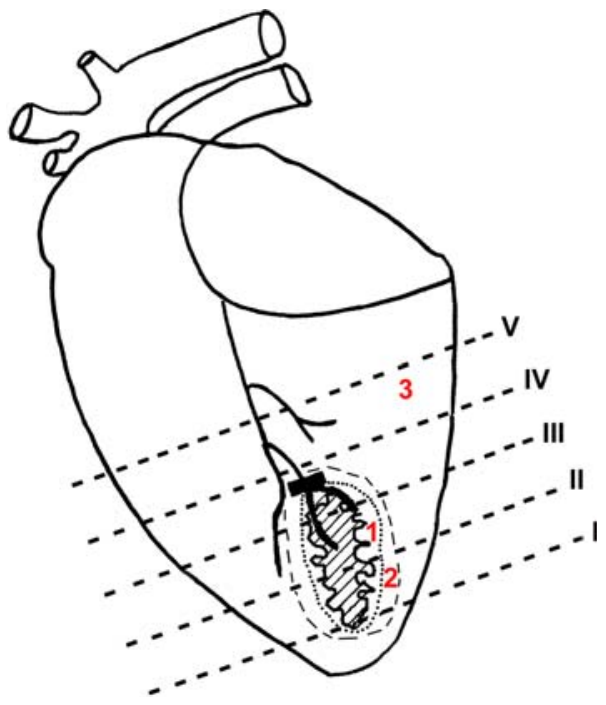

Fig. 1 Macroscopical design of the rabbit LAD ligation model. A suture was placed on a side branch of the left anterior descendens coronary artery (indicated by the black box) leading to an infarction (dashed area) of the underlying left ventricular tissue. 5 consecutive slices (I to V) were made of the rabbit heart immediately after perfusion fixation. Several samples were taken from the border zone of infarcted myocardium (1), from the adjacent non-infarcted region (2) and from the remote zone of the left ventricle (3) 


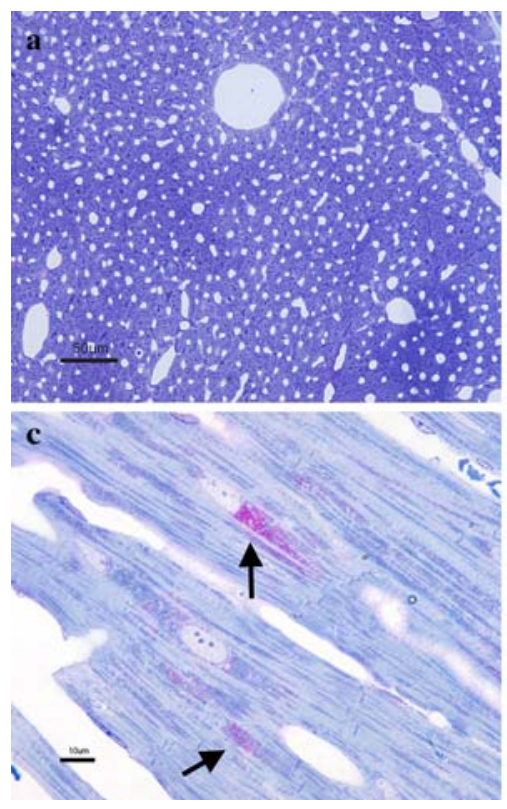

Fig. 2 Light microscopy of periodic acid Schiff and toluidine bluestained sections of rabbit left ventricular tissue. (a) Transverse sections of non-infarcted tissue from a remote zone showing normal distribution of cardiomyocytes and microcirculatory blood vessels. (b) Border zone of infarcted myocardium (1) with the presence of intracellular vacuoles in cardiomyocytes surrounded by fibrotic tissue

\section{Localization of hibernating cardiomyocytes}

In the PAS-stained sections a rather small percentage of CM's, mainly present in the adjacent non-infarcted region, showed some dark pink cytoplasmic staining particularly around the nucleus (Fig. 2c). In the electron microscope these CM's revealed early signs of hibernation i.e. some depletion of sarcomeres in the perinuclear region with concomitant accumulation of glycogen in the cytoplasmic space, a change in mitochondrial ultrastructure characterized by a smaller size and an elongated or donut shape with reorientation of the cristae and a homogeneous distribution of the heterochromatin in the nuclei (Fig. 3b). A low number of CM's in a more advanced stage of hibernation were also seen, but these cells only occurred in the border zone of infarcted myocardium. The cells showed a high degree of myolysis leaving only a few myofibrils at the periphery of the cell, accompanied by a large accumulation of glycogen (Fig. 3c). The number of hibernating CM's was relatively small and similar numbers were seen at different time-periods after ligation.

The presence of vacuolated cardiomyocytes

A remarkable phenomenon of the seemingly isolated, viable CM's in the border zone of infarcted myocardium was the frequent occurrence of intracellular vacuoles

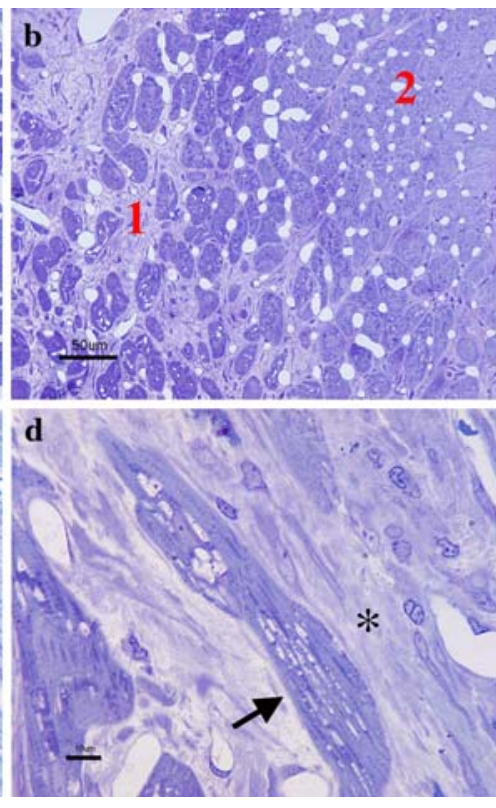

and the adjacent non-infarcted region (2). (c) Longitudinally sectioned hibernating cardiomyocytes with (perinuclear) glycogen accumulation (arrows) within an adjacent non-infarcted region (d) Cardiomyocyte with parallel aligned vacuoles (arrows) and surrounded by fibrotic tissue (asterisk). Bars represent $50 \mu \mathrm{m}(\mathbf{a}, \mathbf{b})$ and $10 \mu \mathrm{m}(\mathbf{c}, \mathbf{d})$, respectively

(Fig. 2b). These vacuoles were often present in parallel arrays in longitudinally sectioned CM's (Fig. 2d), and occurred in comparable frequency and appearance at the different time-periods after LAD ligation. In serial light microscopical sections it was seen that the vacuolated CM's were interconnected with each other and finally with the adjacent non-infarcted myocardium. Electron microscopical analysis showed that most vacuolated CM's contained completely normal sarcomeric structures and mitochondria without any sign of ischemic damage or degeneration (Fig. 4a). Sporadic CM's with structural hallmarks of minor hibernation were seen to contain vacuolar structures as well. Some vacuolated cells revealed lateral irregular protrusions containing myofilaments but no typical sarcomeric apparatus. The vacuoles exhibited a diameter ranging from $200-1,600 \mathrm{~nm}$. In the longitudinally sectioned CM's the parallel arrays of vacuoles were mainly seen at the sarcomeric Z-lines and had a rather similar transverse diameter of about $400 \mathrm{~nm}$ (Fig. 4c). Furthermore, the vacuoles were characterized by the presence of an internal basal lamina (Fig. 4a), by pinocytotic vesicles (Fig. 4b) and by adjoining cisternae of sarcoplasmic reticulum (Fig. 4b). Several vacuoles also contained highly electron dense structures of varying size.

Another peculiar finding was the presence of collagen fibrils in part of the vacuolar structures (Fig. 4c). The number of vacuolated cells positive for collagen fibrils was 

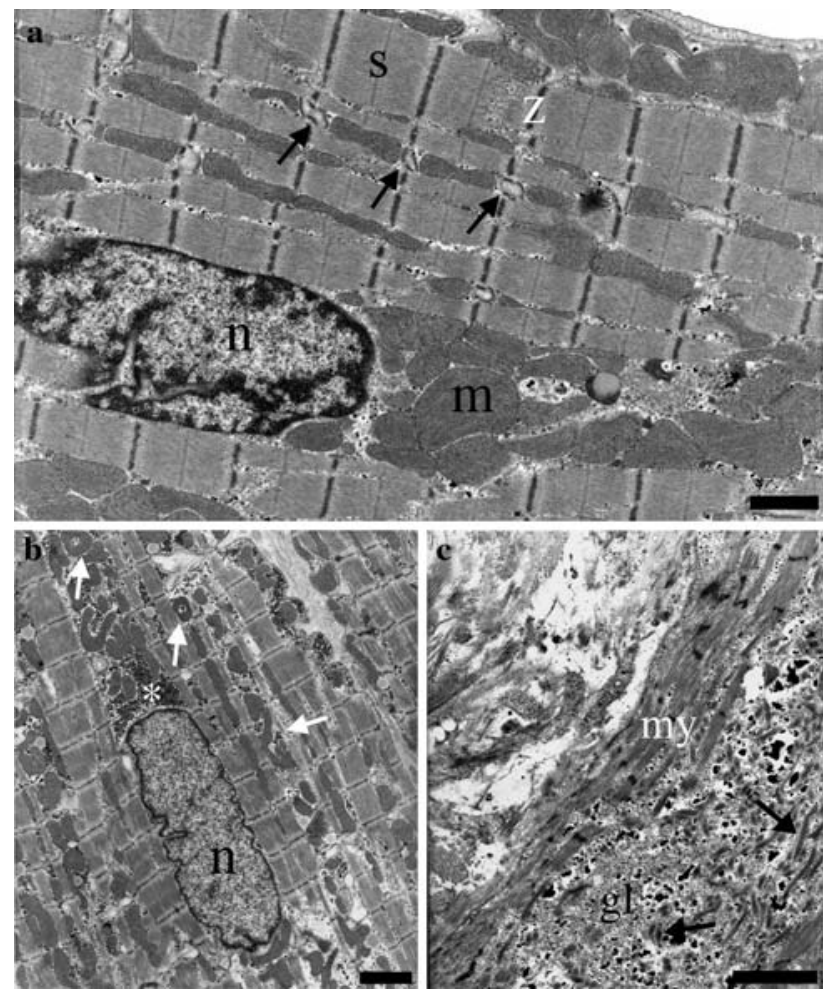

Fig. 3 Electron microscopical pictures of cardiomyocytes in the adjacent non-infarcted myocardium ( $\mathbf{a}$ and $\mathbf{b}$ ) and in the border zone of infarcted myocardium (c). (a) Cardiomyocyte with regularly structured sarcomeres (s) and rows of mitochondria (m). Note the normal appearance and distribution of T-tubules (arrows) at the Zlines (z). (b) Cardiomyocyte with early signs of hibernation showing some distortion of sarcomeric myofilaments and Z-lines, filling of the perinuclear region with glycogen (asterisk), the presence of smaller sized and donut-shaped mitochondria (arrows) and homogeneous distribution of heterochromatin in the nucleus (n). (c) Border zone cardiomyocyte in an advanced stage of hibernation with central depletion of sarcomeres and pronounced accumulation of glycogen (gl). Only a few myofibrils (my) are left at the periphery of the cell. Also note the small, slender mitochondria in the central area (arrows). Bars represent $1 \mu \mathrm{m}(\mathbf{a})$ and $2 \mu \mathrm{m}(\mathbf{b}, \mathbf{c})$, respectively

clearly increased at 8 and 12 weeks after induction of infarction (Fig. 5). Connections of vacuoles with the outer plasma membrane and dilations of intercalated disc areas were sporadically observed.

\section{Morphometric analysis}

The vacuole area/total cell area ratios (\%) of the CM's in the border zone of infarcted myocardium 4, 8 and 12 weeks post-infarction were slightly but significant larger than that after 2 weeks post-infarction $[5.8 \pm 5.6 \% \quad(n=207)$, $6.3 \pm 7.8 \% \quad(n=207), \quad 7.1 \pm 5.3 \quad \% \quad(n=249) \quad v s$ $3.7 \pm 3.3 \% \quad(n=102)$, respectively; $P<0.05$ ] (Fig. 6). Between 4, 8 and 12 weeks post-infarction the ratios were not significantly different.
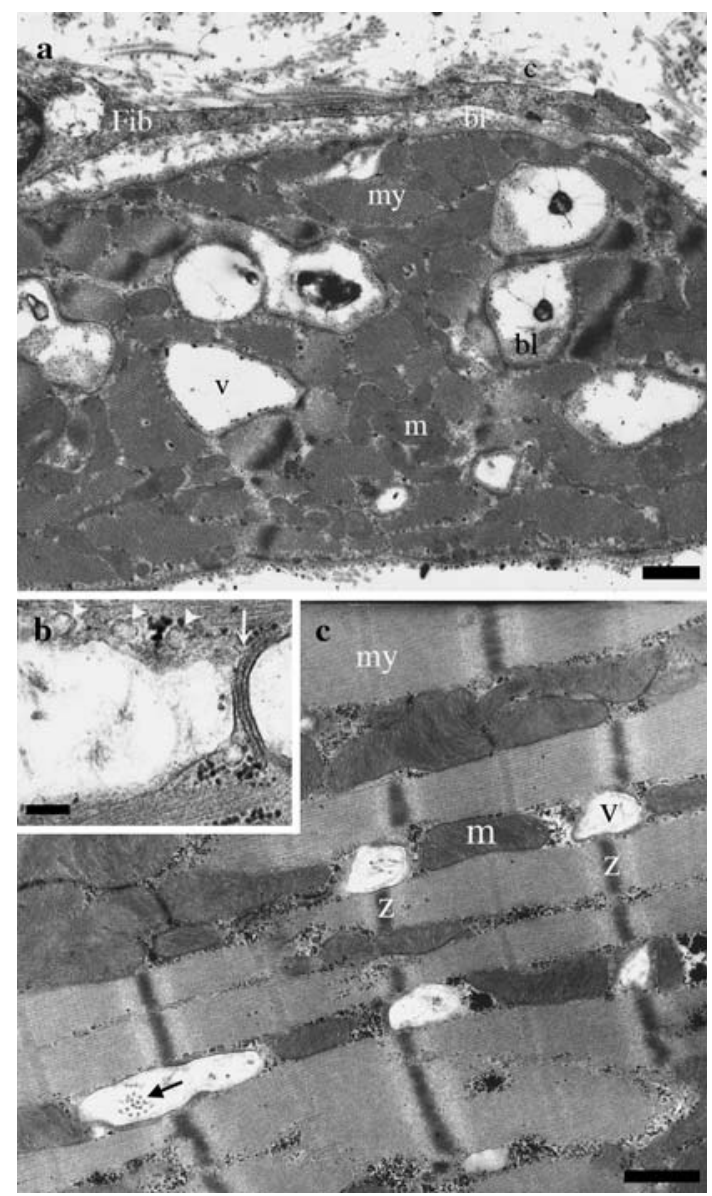

Fig. 4 Electron microscopy of vacuolated cardiomyocytes located in the border zone of infarcted myocardium. (a) Transverse section of a cardiomyocyte containing intracellular vacuoles (v) and intact myofilaments (my) and mitochondria (m). The vacuoles are characterized by an internal basal lamina (black bl) similarly to that at the outer sarcolemmal membrane (white bl). Some vacuoles contain highly electron dense structures. A fibroblast filopodium (Fib) is observed adjacent to the cardiomyocyte. ( $c=$ collagen). (b) Higher magnification of an intracellular vacuole with the presence of pinocytotic vesicles (white arrowheads) and an adjoining cisternum of sarcoplasmic reticulum (white arrow). (c) Longitudinally sectioned cardiomyocyte with parallel-aligned vacuoles (v) at the level of the sarcomeric Z-lines (z) resembling dilated T-tubules. Some vacuoles contain collagen fibrils (arrow). The myofilaments (my) and mitochondria $(\mathrm{m})$ are intact. Samples were taken 1 (a) and 3 months (b and c) after coronary artery ligation. Bars represent $500 \mathrm{~nm}(\mathbf{a}, \mathbf{c})$ and $125 \mathrm{~nm}(\mathbf{b})$, respectively

The cell widths of the CM's in the border zone of infarcted myocardium and in the adjacent non-infarcted myocardium were significantly larger than those in the remote zone in 2 weeks $[20.5 \pm 6.4 \mu \mathrm{m}(n=54)$ and $20.5 \pm 4.6 \mu \mathrm{m} \quad(n=100)$ vs. $16.8 \pm 3.3 \mu \mathrm{m} \quad(n=184)$, respectively; $P<0.05], 4$ weeks $[18.7 \pm 4.8 \mu \mathrm{m}(n=85)$ and $18.6 \pm 4.3 \mu \mathrm{m}(n=152)$ vs. $14.1 \pm 2.8 \mu \mathrm{m}(n=158)$, respectively; $P<0.05], 8$ weeks $[14.7 \pm 3.6 \mu \mathrm{m}(n=72)$ and $18.8 \pm 4.0 \mu \mathrm{m}(n=61)$ vs. $13.5 \pm 2.8 \mu \mathrm{m}(n=127)$, 


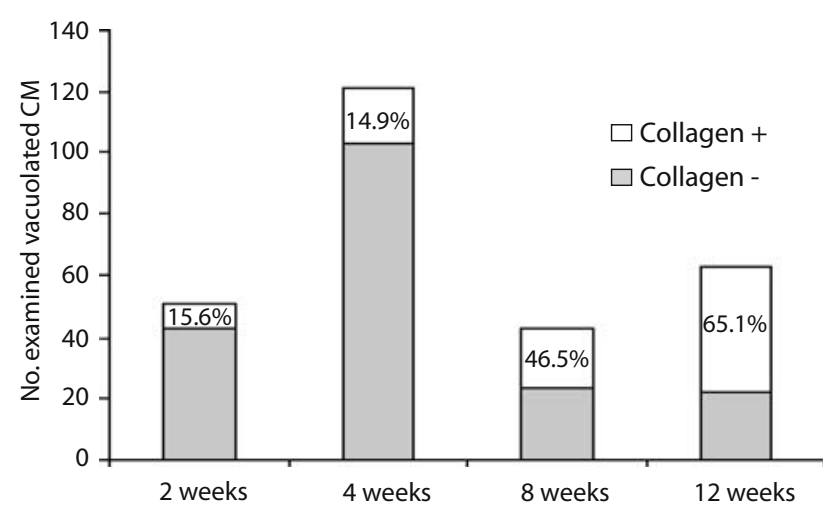

Fig. 5 Presence (collagen + ) or absence (collagen -) of collagen fibrils in vacuolated CM's, as verified by electron microscopy. The number of vacuolated cells containing vacuoles with collagen fibrils is clearly increased at 8 and 12 weeks post-infarction

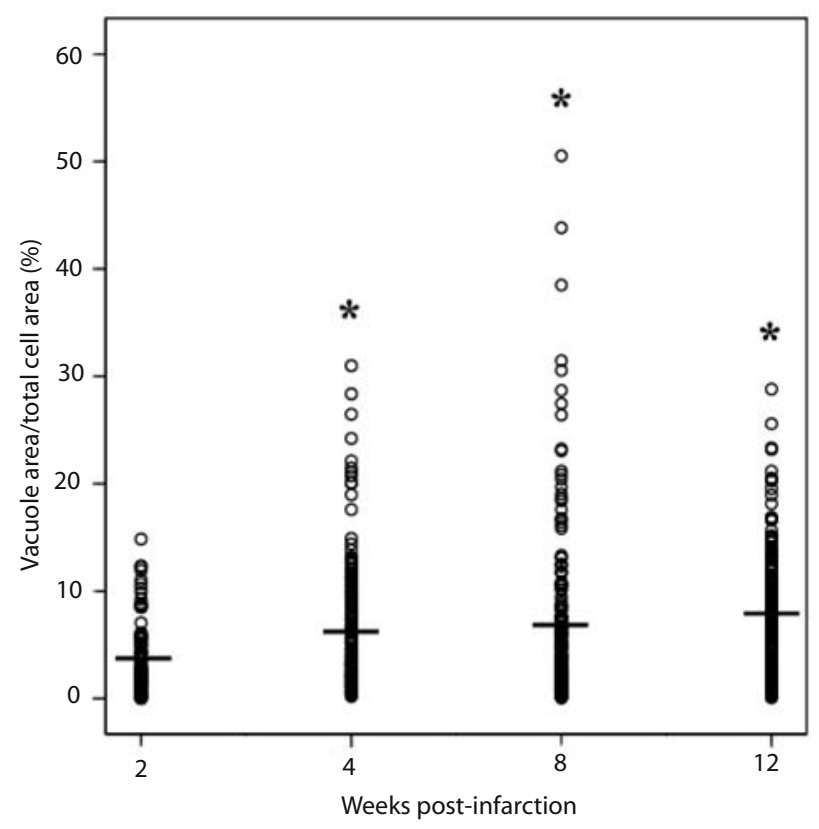

Fig. 6 Light microscopical morphometry: vacuole area/total cell area ratio in myocytes located in the border zone of infarction. Relative to the 2-week period, the ratios at the later time periods were slightly but significantly increased. ${ }^{*} P<0.05$

respectively; $P<0.05]$ and 12 weeks $[18.3 \pm 5.7 \mu \mathrm{m}$ $(n=115)$ and $19.8 \pm 4.1 \mu \mathrm{m}(n=103)$ vs. $14.9 \pm 2.72$ ( $n=194)$, respectively $P<0.05$ ] post-infarction (Fig. 7). At all time-periods after infarction, the cell width of CM's from the adjacent non-infarcted regions were significantly larger than the cell width of CM's located at the corresponding site of the ligated coronary artery in the noninfarcted control heart [0 weeks; $13.3 \pm 2.3 \mu \mathrm{m}(n=200)$, $P<0.05$ ] (Fig. 7).

\section{Discussion}

This study shows that in the time-period between 2 weeks and 3 months after induction of an infarction by coronary artery ligation, dedifferentiated CM's are found in the rabbit heart. Furthermore, within the border zone of infarcted myocardium viable vacuolated cells were observed which were surrounded by fibrotic tissue.

Co-culturing of adult rabbit CM's with cardiac fibroblasts resulted in typical structural characteristics of $\mathrm{CM}$ dedifferentiation, resembling human myocardial hibernation $[3,12,13,16]$. Such hibernating cardiomyocytes have previously been reported at the borders of micro-infarctions induced in sheep after injection of macro-beads into the LAD or CX coronary artery [5] and in regions bordering fibrous scars of dogs with heart failure [4]. Therefore, we speculated that the border zone of infarcted myocardium in the rabbit heart could possibly be used as a small animal model for further studies on hibernating cells. PAS staining indicated that dedifferentiated CM's with the typical morphological characteristics of hibernation were definitely present and that the cells were mainly located in the adjacent non-infarcted region of the infarcted rabbit heart. But, the number of hibernating myocytes appeared too small to be useful for the intended purpose.

The most obvious finding in the border zone of infarcted myocardium, however, was the occurrence of vacuolated cells. This vacuolation was interpreted as unrelated to myocyte degeneration, since the ultrastructural examination revealed completely normal myofibrillar, mitochondrial and other cytoplasmic structures, while also the subcellular distribution of all cytoplasmic organelles was quite normal. Only a few of the vacuolated cells showed minor changes typical for hibernation, indicating that ongoing dedifferentiation is not a prerequisite for the establishment of vacuoles. Furthermore, the vacuolated cells did not resemble the "remnant cardiomyocytes" surrounded by scar tissue described by Sharov et al. [4], since in their study these cells were clearly atrophic. It seems most likely that the vacuolated cells were still under loading condition, as concluded from their mutual interconnection and their connection with the adjacent noninfarcted myocardium. In this way atrophy, as described for unloaded myocardial tissue [4, 17], may have been avoided. Such regions of viable myocardial cells have also been described for dog heart [18]. The irregular protrusions containing non-structured myofilaments found in our material resemble the $\mathrm{CM}$ protrusions described to end in the infarct scar tissue of the rat myocardium [2, 19]. Ultrastructurally, the vacuoles were characterized by a basal lamina closely apposed to its surrounding membrane, and by pinocytotic vesicles at their cytoplasmic side. These characteristics are typical for the sarcolemmal membrane 


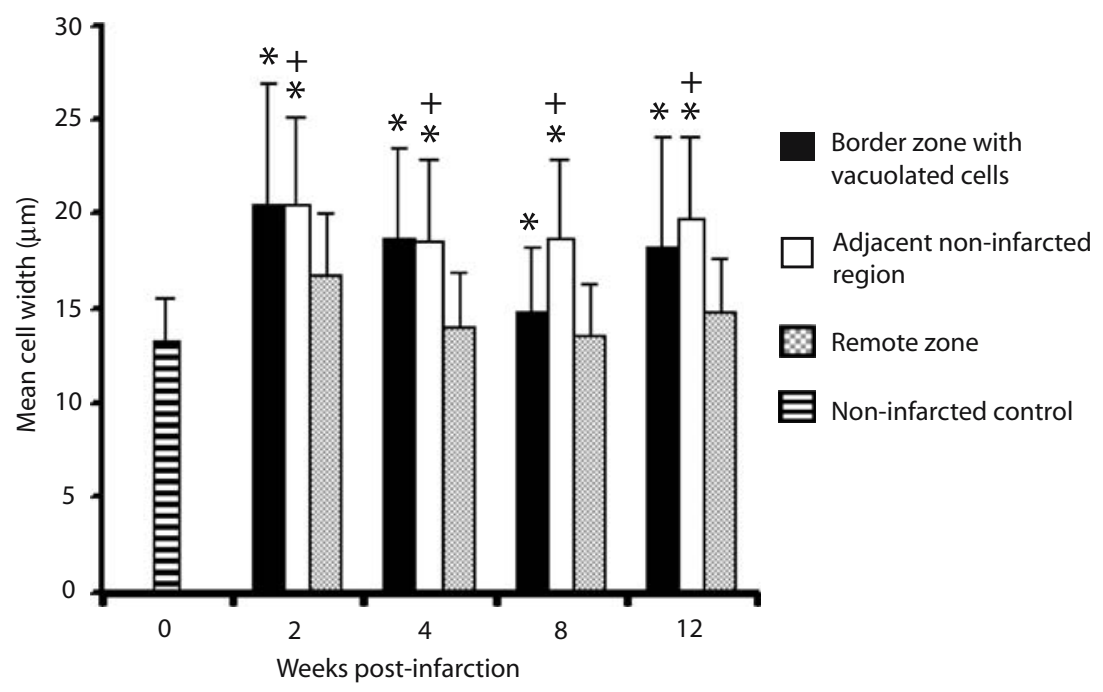

Fig. 7 Mean cell width of CM's located in the border zone, in the adjacent non-infarcted region, in the remote zone of infarcted hearts and in CM's present in a non-infarcted control heart. At the various time-periods after infarction, the mean cell width of vacuolated CM's of the border zone and of the adjacent non-infarcted region is

indicating continuity of the vacuolar membrane with the outer sarcolemma [20]. Furthermore, the vacuoles were seen in close proximity to cisternae of the sarcoplasmic reticulum, which may represent part of a diade structure or of sub-sarcolemmal cisternae. All these characteristics point to a possible duality in the nature of the vacuoles, i.e. they originate from invaginations of the plasma membrane and/or from dilatations of the t-tubular system. The fact that (1) the vacuoles were present in parallel arrangement in longitudinally sectioned CMs, (2) that they were often located at the sarcomeric Z-lines and (3) that they were largely devoid of collagen fibrils at week 2 after LAD ligation, might support the idea of dilation of T-tubules. On the other hand, the fact that some vacuoles were connected to the outer sarcolemmal surface may suggest invagination of the outer sarcolemmal membrane, be it that T-tubules should also be regarded as invaginations.

What remains is the question why these vacuoles develop? The fact that they only occur in CMs in the border zone of infarcted myocardium and are always surrounded by fibrotic scar tissue suggests a mutual relation. Enlarged T-tubules running parallel to the longitudinal axis of the CM's and morphologically resembling the vacuoles seen in our study have been described in human left ventricular tissue from end-stage heart failure secondary to dilated cardiomyopathy [21]. Guinea pigs treated with an overload of volatile anaesthetics have been reported to show dilatations of the T-tubules [22], and dilatation of T-tubules through excessive activation of $\mathrm{Na}^{+}-\mathrm{K}^{+}$-ATPase from the sarcoplasmic side of the T-tubule membrane has been described in transected rat soleus muscle [23]. All these significantly larger than that of CM's from the remote zone indicating myocyte hypertrophy. ${ }^{*} P<0.05$ vs. remote zone. The CM's from the adjacent non-infarcted region have a significant larger width than that of CM's present in the corresponding non-infarcted control region $(0$ weeks $) .{ }^{+} P<0.05$

studies suggest that pathological conditions can result in changes in T-tubule dimensions. In our study, the pathological environment for vacuolization might be created by a limited blood supply and/or the fibrotic surroundings in combination with the (loading) connection with the adjacent non-infarcted myocardium outside the border zone. The fact that the vacuoles were already established within 2 weeks after ligation suggests that they must have been formed at an early stage. After 4 weeks of post-infarction we observed an increase in vacuole area without further significant increment after 8 and 12 weeks post-infarction. Their presence even after 12 weeks is probably related to the persistence of the pathological environment and probably allowed some ongoing remodelling with the surrounding fibrotic tissue as indicated by the progressive increase of collagen within the lumen of these vacuoles. Another adaptive response during cardiac remodelling is the occurrence of myocyte hypertrophy [24]. A significant increase in cell width was measured in vacuolated CM's and in CM's of the adjacent non-infarcted regions in comparison with the CM's of the remote zone. This observation is consistent with the findings of myocyte hypertrophy in non-infarcted myocardium of small animals after coronary ligation $[25,26]$.

In conclusion, this study shows that induction of an infarction by coronary artery ligation in the rabbit heart results in various ways of CM remodelling, i.e. dedifferentiation, vacuolation and hypertrophy. The development of dedifferentiated CM's, with morphological resemblance to hibernation, is rather limited in amount and does not allow to use the ligation procedure as a model system for 
further detailed research. The vacuoles found in viable hypertrophic CM's in the border zone of infarcted myocardium represent dilated T-tubules and/or invaginations of the plasma membrane, and their formation may have been related to the pathologic environment created in the infarcted myocardium.

Acknowledgements We are grateful to Peter Kelderman (Department of Animal Research and Testing Services, University of Maastricht, P.O. Box 616, 6200 MD Maastricht, the Netherlands) for helpful information related to the operation procedures. We thank P.J. Jansen for assistance in statistical analysis.

\section{References}

1. Gottlieb GJ, Kubo SH, Alonso DR (1981) Ultrastructural characterization of the border zone surrounding early experimental myocardial infarcts in dogs. Am J Pathol 103:292-303

2. Matsushita T, Oyamada M, Fujimoto K, Yasuda Y, Masuda S, Wada Y, Oka T, Takamatsu T (1999) Remodeling of cell-cell and cell-extracellular matrix interactions at the border zone of rat myocardial infarcts. Circ Res 85:1046-1055

3. Borgers M, Thoné F, Wouters L, Ausma J, Shivalkar B, Flameng W (1993) Structural correlates of regional myocardial dysfunction in patients with critical coronary stenosis: chronic hibernation? Cardiovasc Pathol 2:237-245

4. Sharov VG, Sabbah HN, Ali AS, Shimoyama H, Lesch M, Goldstein S (1997) Abnormalities of cardiocytes in regions bordering fibrous scars of dogs with heart failure. Int $\mathrm{J}$ Cardiol 60:273-279

5. Dispersyn GD, Mesotten L, Meuris B, Maes A, Mortelmans L, Flameng W, Ramaekers F, Borgers M (2002) Dissociation of cardiomyocyte apoptosis and dedifferentiation in infarct border zones. Eur Heart J 23:849-857

6. Dedkov EI, Stadnikov AA, Russell MW, Borisov AB (2006) Formation of leptofibrils is associated with remodelling of muscle cells and myofibrillogenesis in the border zone of myocardial infarction. Micron DOI 10.1016/j.micron.2006.08.006

7. Rahimtoola SH (1989) The hibernating myocardium. Am Heart J 117:211-221

8. Kloner RA, Bolli R, Marban E, Reinlib L, Braunwald E (1998) Medical and cellular implications of stunning, hibernation, and preconditioning. An NHLBI Workshop. Circulation 97: 1848-1867

9. Maes A, Flameng W, Nuyts J, Borgers M, Shivalkar B, Ausma J, Bormans G, Schiepers C, De Roo M, Mortelmans L (1994) Histological alterations in chronically hypoperfused myocardium. Correlation with PET findings. Circulation 90:735-745

10. Vanoverschelde JL, Wijns W, Borgers M, Heyndrickx G, Depré C, Flameng W, Melin JA (1997) Chronic myocardial hibernation in humans. From bedside to bench. Circulation 95:1961-1971

11. Vanoverschelde JL, Depré C, Gerber BL, Borgers M, Wijns W, Robert A, Dion R, Melin JA (2000) Time course of functional recovery after coronary artery bypass graft surgery in patients with chronic left ventricular ischemic dysfunction. Am J Cardiol 85:1432-1439

12. Dispersyn GD, Geuens E, Ver Donck L, Ramaekers FCS, Borgers M (2001) Adult rabbit cardiomyocytes undergo hibernation-like dedifferentiation when co-cultured with cardiac fibroblasts. Cardiovasc Res 51:230-240

13. Driesen RB, Verheyen FK, Dispersyn GD, Thoné F, Lenders M-H, Ramaekers FCS, Borgers M (2006) Structural adaptation in adult rabbit ventricular myocytes. Influence of dynamic physical interaction with fibroblasts. Cell Biochem Biophys 44:119-128

14. Verdouw PD, van den Doel MA, Zeeuw S, Duncker DJ (1998) Animal models in the study of myocardial ischemia and ischaemic syndromes. Cardiovasc Res 39:121-135

15. Morales C, Gonzalez GE, Rodriguez M, Bertolasi CA, Gelpi RJ (2002) Histopathologic time course of myocardial infarct in rabbit hearts. Cardiovasc Pathol 11:339-345

16. Driesen RB, Dispersyn GD, Verheyen FK, van den Eijnde SM, Hofstra L, Thoné F, Dijkstra P, Debie W, Borgers M, Ramaekers FCS (2005) Partial cell fusion: a newly recognized type of communication between dedifferentiating cardiomyocytes and fibroblasts. Cardiovasc Res 68:37-46

17. Cooper GT, Tomanek R J (1982) Load regulation of the structure, composition, and function of mammalian myocardium. Circ Res 50:788-798

18. Factor SM, Sonnenblick EH, Kirk ES (1978) The histologic border zone of acute myocardial infarction-islands or peninsulas? Am J Pathol 92:111-124

19. Vracko R, Thorning D, Frederickson RG, Cunningham D (1988) Myocyte reactions at the borders of injured and healing rat myocardium. Lab Invest 59:104-114

20. Avettey AS, Navaratnam V (1978) The T-tubule system in the specialized and general myocardium of the rat. J Anat 127: $125-140$

21. Kaprielian RR, Stevenson S, Rothery SM, Cullen MJ, Severs NJ (2000) Distinct patterns of dystrophin organization in myocyte sarcolemma and transverse tubules of normal and diseased human myocardium. Circulation 101:2586-2594

22. Döring HJ (1975) Reversible and irreversible forms of contractile failure caused by disturbances by general anesthetics in myocardial ATP utilization. Recent Adv Stud Cardiac Struct Metab 5:395-403

23. Casademont J, Carpenter S, Karpati G (1988) Vacuolation of muscle fibers near sarcolemmal breaks represents T-tubule dilatation secondary to enhanced sodium pump activity. J Neuropathol Exp Neurol 47:618-628

24. Sutton MG, Sharpe N (2000) Left ventricular remodeling after myocardial infarction: pathophysiology and therapy. Circulation 101:2981-2988

25. Janssens S, Pokreisz P, Schoonjans L, Pellens M, Vermeersch P, Tjwa M, Jans P, Scherrer-Crosbie M, Picard MH, Szelid Z, Gillijns H, Van de Werf F, Collen D, Bloch KD (2004) Cardiomyocyte-specific overexpression of nitric oxide synthase $3 \mathrm{im}$ proves left ventricular performance and reduces compensatory hypertrophy after myocardial infarction. Circ Res 94:1256-1262

26. Surber R, Bollensdorff C, Betge S, Zimmer T, Benndorf K (2006) $\mathrm{K}$ (ATP) channel current increases in postinfarction remodeled cardiomyocytes. Pflugers Arch 452:428-434 